\title{
Vom Öko- zum Nachhaltigkeitsfonds
}

\author{
Immer mehr Menschen kennen und interessieren sich für ethische Anlagepro- \\ dukte. Dennoch klafft zwischen Interesse, bekundeten Verhaltensabsichten und \\ dem tatsächlichen Verhalten häufig noch eine deutliche Lücke. Eine repräsenta- \\ tive Umfrage hat nun wesentliche Hemmnisse und Unsicherheiten identifiziert, \\ die einer größeren Verbreitung im Wege stehen.
}

$\mathrm{D}$ Von Kathrin Klaffke und Roland Pätzold ie Bekanntheit und das Interesse an ethisch-ökologischer Geldanlage in Deutschland wächst. Dies ergab eine repräsentative Befragung privater Anleger, die vom imug Institut für Markt - Umwelt - Gesellschaft e.V. und dem Lehrstuhl für Markt und Konsum der Universität Hannover Anfang 2001 durchgeführt wurde. Bei den telefonisch durchgeführten Interviews wurden 1.204 Bürgerinnen und Bürger befragt, die an den Anlageentscheidungen des jeweiligen Haushalts mitwirken.

Während in früheren Untersuchungen das Interesse an sozial-ökologischen Geldanlagen bei 26 Prozent lag, gaben dies in der neueren Erhebung 44 Prozent der Befragten an. Dass jedoch zwischen Interesse, bekundeten Verhaltensabsichten und dem tatsächlichen Verhalten häufig eine deutliche Lücke klafft, zeigt auch diese Untersuchung. Denn nur drei Prozent der Befragten, die bereits von ethischen Fonds gehört hatten, gaben an, dass ihnen schon einmal ein sozial-ökologischer Wertpapierfonds angeboten worden ist. Und nur 0,68 Prozent dieser Gruppe haben tatsächlich in einen solchen Fonds investiert.

Häufig wurde als Grund angegeben, dass den Befragten unbekannt war, wo solche Fonds erhältlich sind und wo zuverlässige Informationen zu erhalten sind (vgl. Abb. 1). So gaben über 40 Prozent der Befragten, die sozial-ökologische Geldanlagen kennen, aber noch kein Geld in solche Anlagen investiert haben, an, dass es schwierig sei, überhaupt zuverlässige Informationen zu diesen Fonds zu bekommen. Und mehr als einem Drittel der Befragten war es gänzlich unbekannt, woher man diese Fonds bekommt. Dies lässt auf einen hohen Kommunikations- und Informationsbedarf schließen. Um dieses Defizit zu beheben, ist es erforderlich, dass Finanzdienstleister das Angebot an sozial-ökologischen Fonds besser präsentieren und ihr Personal mit dem neuen Aspekt der Geldanlage vertraut machen.
So müssen denn auch die Ergebnisse der imug/ muk-Befragung dahingehend interpretiert werden, dass sich der Markt für sozial-ökologische Geldanlagen bei den privaten Investoren noch in der Aufbauphase befindet. Zwar können sich viele Anleger durchaus vorstellen, in diesem Sektor zu investieren, die allgemein bekundete Bereitschaft sich für diese Form der Kapitalanlage zu entscheiden, muss jedoch wegen der bestehenden Hemmnisse und Unsicherheiten relativiert werden.

Neben den erwähnten Unsicherheiten bezüglich der Glaubwürdigkeit und der Informationsverbreitung spielen nämlich die alten Auswahlkriterien für die Kapitalanlage eine wesentlich bedeutendere Rolle. Denn nach wie vor stehen Risiko- und Renditeaspekte im Mittelpunkt der Überlegung, wenn sich private Anleger dafür entscheiden, ihr Geld am Kapitalmarkt in Aktien oder Wertpapierfonds anzulegen. Da nach wie vor nur wenige Anleger über Erfahrungen mit diesen Pro- dukten verfügen, müssen zunächst die bestehenden Vorbehalte gegenüber diesen Produkten abgebaut und der Umgang mit ihnen eingeübt und normalisiert werden.

\section{Riester-Rente als Chance}

Interessanterweise bietet sich gerade jetzt die Möglichkeit, die beiden vorgenannten Problembereiche als eine gemeinsame Herausforderung zu betrachten und diese durch die Entwicklung entsprechender Strukturen und Produkte auch zu meistern. Gemeint sind hier die ab dem neuen Jahr gültigen Veränderungen bei der betrieblichen und privaten Altersvorsorge. Im Rahmen der Gesetzesänderungen wird unter anderem die Option eingeräumt, Kapitalmittel in Pensionsfonds einzubringen, um so für den Lebensabend vorzusorgen. Gleichzeitig sind die entsprechenden Fondsanbieter gesetzlich dazu verpflichtet, anzugeben, ob und wie sie ethische, soziale und ökologische Belange bei der Verwendung der eingezahlten Beiträge berücksichtigen. Dies ermöglicht, die privaten Anleger mit Fondsprodukten vertraut zu machen und gleichzeitig bei der Auswahl der im Fonds vertretenen Unternehmen die Grundlagen des ethischen Investments umzusetzen.

Von Vorteil ist dabei, dass sich der Staat und die Unternehmen ebenfalls in der Rolle der Promotoren befinden und dadurch eine höhere Glaubwürdigkeit erzielt wird, als wenn ausschließlich die Finanzdienstleister diese Produkte vermarkten würden. Und Einrichtungen wie etwa die Verbrau-

\section{Kein Geld in sozial-ökologischen Fonds angelegt, ... Warum haben Sie bisher kein Geld in sozial-ökologischen Fonds angelegt?}

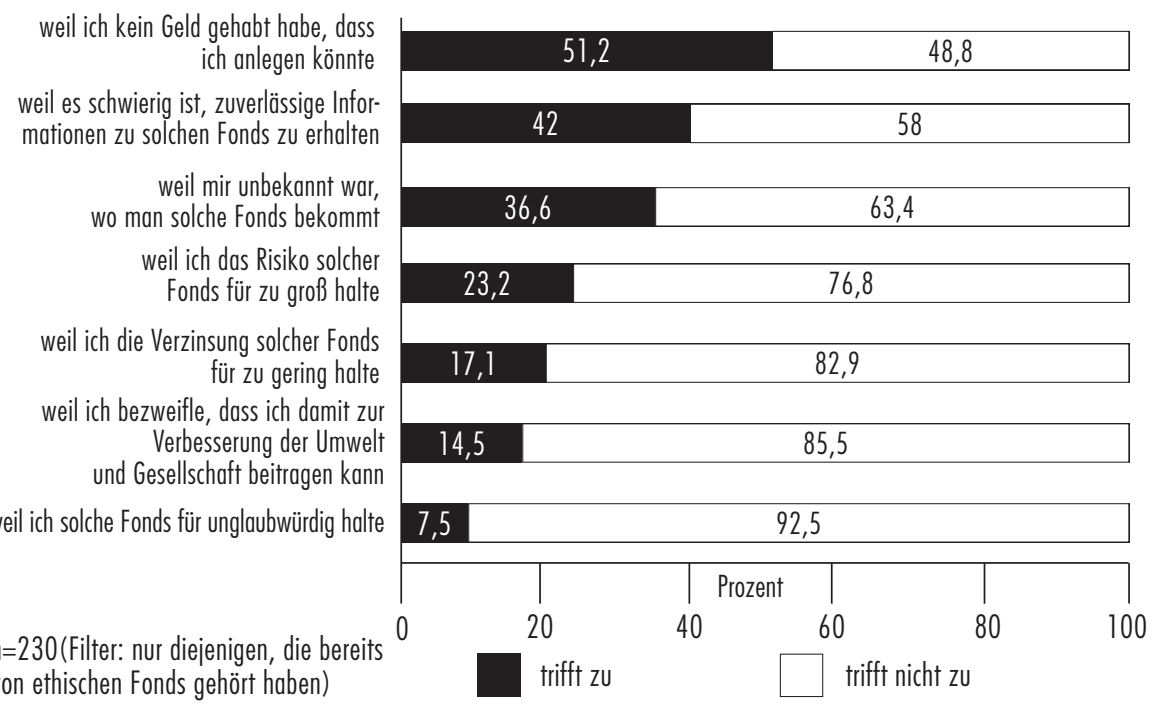


D Von Christian Kolarik, Liselotte Ledvonova und Martina Strassmai

e Anlage in ethisch-ökologisch orientierte Fonds ist seit längerem ein Thema in Deutschland (vgl. den Beitrag von Klaffke/Pätzold) und der Schweiz. In Österreich handelt es sich zwar auch um ein aufstrebendes Segment, Skepsis ist jedoch nach wie vor vorhanden. Dies ergab eine Expertenumfrage, die bei den Fondsmanagern von 24 Kapitalanlagegesellschaften (KAG) in Österreich im Frühjahr 2001 vom Institut für Betriebliche und Regionale Umweltwirtschaft an der Johannes Kepler Universität Linz durchgeführt wurde. Zu Beginn diesen Jahres gab es nur einen einzigen rein österreichischen ethisch-ökologischen Fonds, mittlerweile werden weitere angeboten. Der bedeutende potenzielle Einfluss des Finanzdienstleistungssektors auf Umwelt und Soziales wird von der Mehrzahl der Befragten gravierend unterschätzt. Zwei Drittel räumten nur einen mittleren bis geringen Einfluss ein. Die Bewertung der ethisch-ökologischen Fonds durch die KAGs hinsichtlich der konventionellen ökonomischen Parameter Risiko, Rendite, Sicherheit und Liquidität fiel relativ identisch mit der Bewertung herkömmlicher Fonds aus. Die Nachfrage im Vergleich zu herkömmlichen Fonds bewerten jedoch über 80 Prozent als eindeutig schlechter. Die Ursachen für das noch geringe Angebot dürften unter anderem mit negativen Erfahrungen aus Bemühungen zu Beginn der 90 er Jahre zusammenhängen. Von den damals lancierten Fonds mussten alle mangels Nachfrage wieder eingestellt werden. Auch rechnet über die Hälfte der befragten KAGs mit einem Anteil von höchstens fünf Prozent an Privatanlegern, die in Österreich an ethisch-
Ergebnisse zweier Studien zu ökologischen Anlageprodukten in Österreich

\section{Ignorante Finanzdienstleister?}

Finanzdienstleister bestimmen zukünftige Umweltbeund -entlastungen maßgeblich mit. Im Rahmen zweier

Befragungen wurden die Aktivitäten von Fondsmanagern und Versicherungen auf dem österreichischen Markt genauer beleuchtet.

ökologischen Geldanlageformen interessiert sein könnten. Im Gegensatz dazu gaben in einer Gallup-Studie aus dem Jahre 199742 Prozent der an „höherer" Veranlagung Interessierten an, dass sie bei der Geldanlage soziale bzw. ökologische Aspekte berücksichtigen würden.

\section{Versicherungsgesellschaften als Nachfragestimulatoren?}

Um die mangelnde Nachfrage nach ethisch-ökologischen Produkten zu stimulieren, hofft man gerade in einem ersten Schritt auf die institutionellen Anleger, wie sie etwa in Form der Kapitalveranlagung der Versicherungen bestehen. Eine Befragung unter den 54 am österreichischen Markt tätigen Versicherungsgesellschaften ergab, dass Produkte im Sinne von Versicherungsangeboten mit ökologieorientierten Komponenten derzeit nur sehr vereinzelt angeboten werden. Diese beziehen sich unter anderem auf Ermäßigungen bei der Krafffahrzeugversicherung, die an Kunden gewährt werden, die im Besitz einer Jahreskarte für das öffentliche Verkehrsnetz sind. Im Bereich Lebens- und Pensionsversicherungen wurden hingegen von den befragten Versicherungen keine fondsgebundenen Alternativen genannt, die akzentuiert auf ökologische oder ethische Aspekte ausgerichtet sind.

Auch gab keine der Gesellschaften an, im Rahmen der Vermögensverwaltung einen festgelegten Anteil des Kapitals in Fonds mit ökologischer und/oder ethischer Ausrichtung anzulegen. Aspekte der Umwelt und Ethik werden nicht als explizite Kriterien oder als spezifische Anlagestrategie bei Fondsentscheidungen verfolgt. Zusammenfassend lässt sich feststellen, das auf Seite der institutionellen Nachfrager ein an sich vorhandenes erhebliches Potenzial bisher noch weitgehend ungenutzt ist. Ein abgestimmtes Vorgehen des Akteursdreieckes Banken Kapitalanlagegesellschaften - Versicherungen, sowie eine entsprechende Sensibilisierung, könnte hier den Beginn eines möglichen Strukturwandels einleiten.

Die AutorInnen
Christian Kolarik ist wissenschaftlicher Assistent,
Liselotte Ledvonova und Martina Strassmair sind
Diplomanden am Institut für Betriebliche und
Regionale Umweltwirtschaft der Johannes Kepler
Universität Linz
Kontakt: Universität Linz, Altenbergerstr. 69,
A-4040 Linz, Tel. 0043-732-2468-8833,
Fax -8832, E-mail: office@oek0.uni-linz.ac.at

cherzentralen werden dafür sorgen, dass deutlich wird, welche Finanzprodukte das sozial-ökologische Unbedenklichkeitssiegel zu Recht tragen (vgl. dazu auch den Kommentar auf S.4).

Da Fondsmanager künftig bei der Ausgestaltung der sozial-ökologischen Produkte immer auf eine Auswahl von Positiv- bzw. Negativkriterien zurückgreifen werden, ist es notwendig die Präferenzen der möglichen Kunden zu kennen. Detaillierte Betrachtungen unterschiedlicher Anlegergruppen liefern Ansatzpunkte für eine zielgruppenspezifische Marketingstrategie (1). Mit diesen Kenntnissen können Fonds in Zukunft besser auf potenzielle Anleger im Marktsegment ethisches Investment abgestimmt werden.

\section{Große Zielgruppe}

Für rund 37 Prozent der Befragten ist das sozialökologische Engagement der Unternehmen ein wichtiges Auswahlkriterium. Von einem Finanz- produkt erwarten sie neben einer wenigstens durchschnittlichen Rendite, dass außer den üblichen Ausschlusskriterien Waffenproduktion, Kinderarbeit, etc. in Zukunft auch vermehrt Positivkriterien wie Umweltschutz oder Informationsoffenheit berücksichtigt werden. Darüber hinaus gibt es aber auch Kriterien, die je nach Ausrichtung und möglicher Kundengruppe Berücksichtigung finden werden. Hierzu könnten zum Beispiel Gentechnik und Tierversuche zählen.

Außerdem ist für diese Zielgruppe das Interesse an den sozialen Kriterien besonders ausgeprägt. Vermutlich wird es zukünftig also nicht mehr ausreichen, Unternehmen nur nach ihrem ökologischen Engagement zu bewerten. Auch die sozialen Leistungen der Unternehmen sind $\mathrm{zu}$ überprüfen und als Auswahlkriterium einzubinden. Deshalb kann der Schluss gezogen werden, dass die Anleger in Zukunft nicht mehr nur an reinen Ökofonds, sondern vielmehr an Nachhaltigkeitsfonds interessiert sind.

\section{Anmerkung}

(1) Vgl. hierzu ausführlicher imug/muk (Hrsg.): Der Markt für sozial-ökologische Geldanlagen in Deutschland. imug-Arbeitspapier 13/2001, Hannover.

Die AutorInnen
Kathrin Klaffke und Roland Pätzold sind
wissenschaftliche MitarbeiterInnen des
Instituts für Markt-Umwelt-Gesellschaft
an der Universität Hannover (imug).
Kontakt: imug e.V., Escherstr. 23, 30159
Hannover, Tel.0511/ 91115-17, Fax -95,
E-Mail: klaffke@imug.de


(c) 20I0 Authors; licensee IÖW and oekom verlag. This is an article distributed under the terms of the Creative Commons Attribution Non-Commercial No Derivates License (http://creativecommons.org/licenses/by-nc-nd/3.o/), which permits unrestricted use, distribution, and reproduction in any medium, provided the original work is properly cited. 